\title{
EFFECTS OF ADDITIVE ON THE MECHANICAL PROPERTIES OF BAMBOO/PBS COMPOSITES
}

\author{
YEON-HEE LEE ${ }^{\dagger}$ HAN-KI YOON \\ Department of Mechanical Engineering, Dong-Eui University, Busanjin-Gu, Busan, 614-714, Korea \\ nalrasuk@hanmail.net,hkyoon@deu.ac \\ HITOSHI TAKAGI \\ Institute of Technology and Science, The University of Tokushima 2-1 Minamijosanjima-cho, \\ Tokushima 770-8506, Japan \\ takagi@me.tokushima-u.ac.jp ${ }^{1}$ \\ KAZUYA OHKITA \\ In Wood Processing Section, Kagawa Prefectural Industrial Technology Center587-1 Goto-cho, \\ Takamatsu 761-8031, Japan \\ vm8523@pref.kagawa.lg.jp ${ }^{3}$
}

\begin{abstract}
Compared with general composites which are produced from fossil fuel, biodegradable resins have received considerable attention as an environment-friendly material. Bamboo fiber has relatively high strength compared with other natural fibers. Therefore, the focus of this study is to produce bamboo fiber reinforced Poly butylene succinate (PBS) composites by injection molding and to study the effects of additive on mechanical properties of this bamboo/PBS composite. The injectionmolding is a highly productive fabrication technique. Bamboo/PBS composites were examined by flexural test and Vickers hardness. Also we examined fracture surface and microstructure of the bamboo/PBS composites by microscope.
\end{abstract}

Keywords: Green composites, Bamboo fiber, Flexural strength, Vickers hardness

\section{Introduction}

Natural fibers have recently attracted the attention of many researchers because of the advantages that these fibers provide over conventional reinforcement materials, and the development of natural fiber composites has been a subject of interest for the past few years. Research on the green composite, which are composed biodegradable resins and natural fibers such as ramie, hemp, pineapple, henequen and bamboo, has been carried out $^{1,2}$. Bamboo and kenaf has been already adopted as automobile parts, because of it has lightweight and good mechanical properties among other natural fibers. Also biodegradable resins receive considerable attention as environment-friendly material because biodegradable resins may be resolved in both water and carbon dioxide after 
perfect biodegradation by the action of microorganisms ${ }^{2}$. But almost of biodegradable resins have relatively low strength than typical resins such as polyethylene, it is impossible to use them as structures material. Accordingly, research on the green composite, which are composed biodegradable resins and natural fibers has been carried out. PBS is a white crystalline thermoplastic with melting point of about $90^{\circ} \mathrm{C}$ to $1208^{\circ} \mathrm{C}$ (similar to LDPE), glass transition temperature of about $45^{\circ} \mathrm{C}$ to $108^{\circ} \mathrm{C}$ (between $\mathrm{PE}$ and PP), tensile strength between PE and PP, and stiffness between LDPE and HDPE. PBS has excellent processing capabilities and can be processed on polyolefin processing machines at temperatures of $160^{\circ} \mathrm{C}$ to $2008^{\circ} \mathrm{C}$, into various products, such as infected, extruded and blown ones ${ }^{3}$. Furthermore, its raw materials, butanediol and succinic acid, may be soon available from bio-based renewable resources ${ }^{4-9}$.

In this study, we have carried out research on effects of additive on mechanical properties of bamboo/PBS composites. By using additive with two kinds of type and two kinds of amount, bamboo fibers with 40wt.\% content and the biodegradable resin (PBS 60wt.\%) were produced by hot-pressing. PVA (polyvinyl alcohol) and CMC (carboxy methylcellulose) were used as an additive. PVA has excellent film forming, emulsifying, and adhesive properties. It is also resistant to oil, grease and solvent. It is odorless and nontoxic. It has high tensile strength and flexibility, as well as high oxygen and aroma barrier properties. However these properties are dependent on humidity, in other words, with higher humidity more water is absorbed. The water, which acts as a plasticiser, will then reduce its tensile strength, but increase its elongation and tear strength. PVA is fully degradable and is a quick dissolver. CMC is a cellulose derivative with carboxymethyl groups $(-\mathrm{CH} 2-\mathrm{COOH})$ bound to some of the hydroxyl groups of the glucopyranose monomers that make up the cellulose backbone. It is often used as its sodium salt, sodium carboxymethyl cellulose.

\section{Experiment}

\subsection{Sample Preparation}

Bamboo/PBS composites were fabricated, using a length $-250 \mu \mathrm{m}$ to $+500 \mu \mathrm{m}$ short bamboo fiber that were dried for 24 hour at $105^{\circ} \mathrm{C}$ with a fiber content of $40 . \mathrm{wt} \%$. Where $(-)$ means that fibers passed through the mesh, and (+) means that fibers were retained on the mesh. The bamboo fibers were used as received conditions without any surface treatment. The PBS (polybutylene succinate) resin (Showa Highpolymer Co., Ltd., Japan) was used as a matrix resin. The additives were used PVA and CMC $(12.5 \mathrm{~g}$ and $25 \mathrm{~g}$ respectively). Bamboo fiber and PBS were thrown into an extruder (TITAN45, Cincinnati Extrusion GmbH, Austria) to make bamboo/PBS pellets. The bamboo/PBS pellets was hot-pressed under a pressure of $10 \mathrm{MPa}$ at $130^{\circ} \mathrm{C}$ for $4 \mathrm{~min}$. 


\subsection{Measurement of mechanical properties and Surface characterization}

The flexural strength was measured using a 3-point bending tests using a universal testing machine with a specimen size of $50 \mathrm{~mm} \times 3.8 \mathrm{~mm} \times 5 \mathrm{~mm}$, crosshead speed of $0.5 \mathrm{~mm} / \mathrm{min}$ and a span length of $30 \mathrm{~mm}$ at room temperature, using the four specimens.

Vickers hardness of bamboo/PBS composite was investigated by using a Mitutoyo Hardness testing machine. We also observed the fracture surface of bamboo/PBS composite after 3-point bending tests by field emission scanning electron microscopy (FE-SEM).

\section{Experimental Results and Discussion}

Fig. 1 (a) shows the flexural strength from 3-point bending test of the bamboo/PBS composite using without additive, CMC $12.5 \mathrm{~g}$ and $\mathrm{CMC} 25 \mathrm{~g}$. Without additive bamboo/PBS composites possessed an average flexural strength of about $42.87 \mathrm{MPa}$, which lower than that CMC $12.5 \mathrm{~g}$ (about 44.05MPa). Fig. 1 (b) shows the flexural strength of bamboo/PBS composites using PVA (without additive, $12.5 \mathrm{~g}$ and $25 \mathrm{~g}$ ). The result showed $44.08 \mathrm{MPa}$ at PVA $12.5 \mathrm{~g}$, the minimum value of $35.24 \mathrm{MPa}$ at PVA $25 \mathrm{~g}$. Fig. 2 shows the fracture surface of bamboo/PBS composites without additive, CMC $12.5 \mathrm{~g}$ and PVA $12.5 \mathrm{~g}$. In the case of bamboo/PBS composites without additive, it can be seen from this figure that there is many pull-outed fiber on the fracture surface, which is indicating weak adhesion between bamboo fiber and PBS resin. However we can be seen less pull-outed fiber and sticky case of CMC 12.5g and PVA $12.5 \mathrm{~g}$. Therefore, bamboo/PBS composites with CMC 12.5g and PVA $12.5 \mathrm{~g}$ shows higher than flexural strength of without additive.
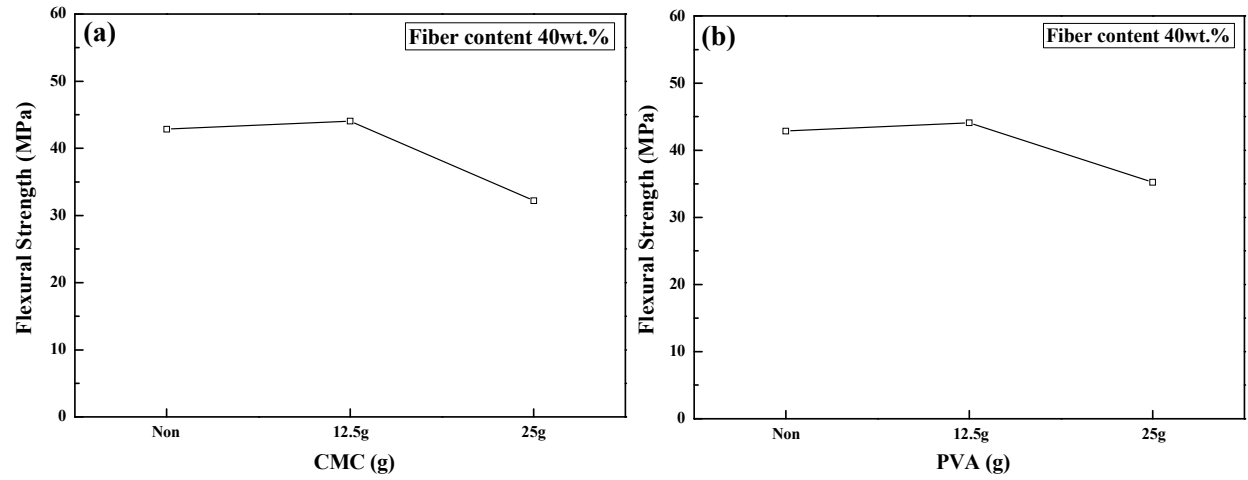

Fig. 1 Effect of additive on flexural strength of bamboo/PBS composites 

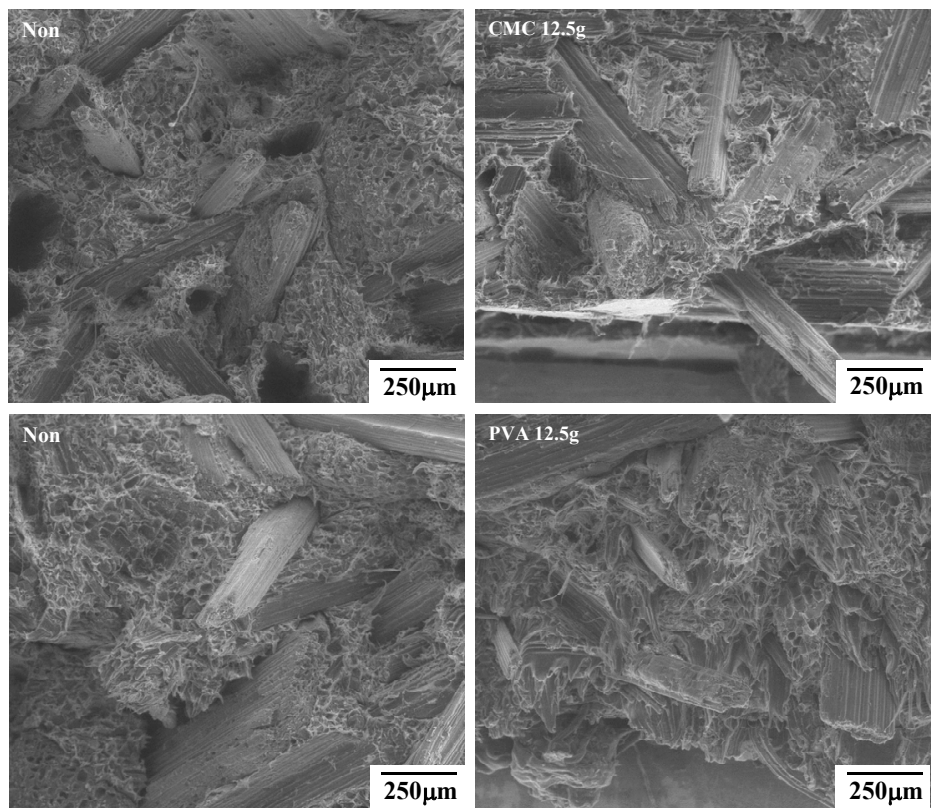

Fig. 2 Fracture surface of bamboo/PBS composites after 3-point bending test

The effect of additive on Vickers hardness of bamboo/PBS composites are shown in Fig. 3. In case of used CMC additive, the maximum value of $35.1 \mathrm{HV}$ of CMC $12.5 \mathrm{~g}$ (Fig. 3(a)). Fig. 3(b) showed Vickers hardness of bamboo/PBS composites used PVA additive. It can be seen highest Vickers hardness value case of $12.5 \mathrm{~g}$.
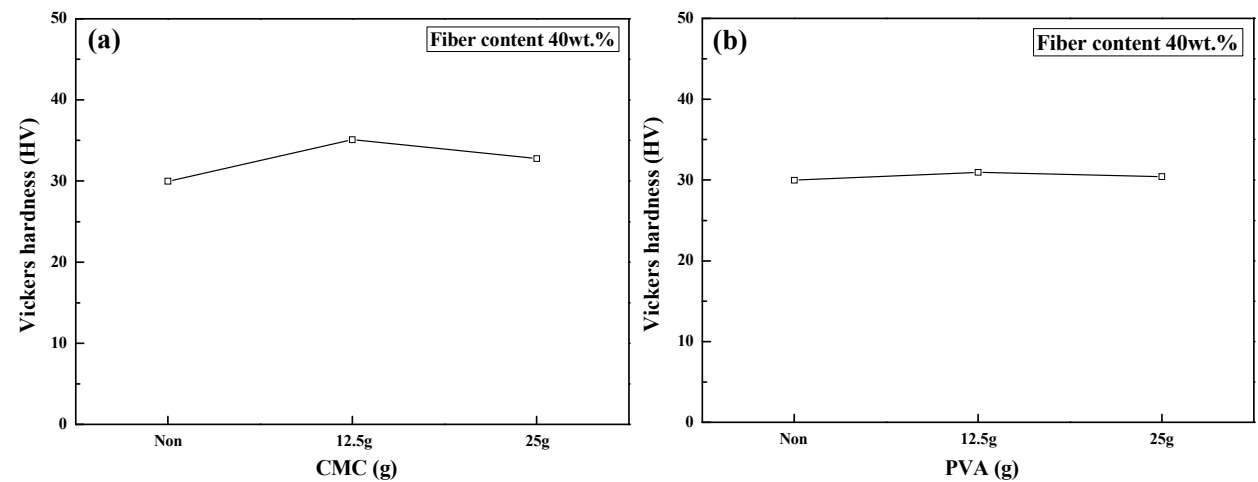

Fig. 3 Effect of additive on Vickers hardness of bamboo/PBS composites 


\section{Conclusions}

In this study, we fabricated bamboo fiber reinforced PBS composites with additive, and evaluated their mechanical properties such as flexural strength, Vickers hardness. The results obtained are summarized as follows,

(1) The effect of additive on flexural strength showed $42.87 \mathrm{MPa}$ at without additive, $44.05 \mathrm{MPa}$ at $\mathrm{CMC} 12.5 \mathrm{~g}$ and $32.19 \mathrm{MPa}$ at $\mathrm{CMC} 25 \mathrm{~g}$. In case of PVA additive, that showed $42.87 \mathrm{MPa}$ at without additive, $44.08 \mathrm{MPa}$ at $12.5 \mathrm{~g}$ and $35.24 \mathrm{MPa}$ at $25 \mathrm{~g}$. (2) The fracture surface of the bamboo/PBS composites with additive CMC 12.5g and PVA 12.5 showed less pull-outed fiber and sticky. Therefore, bamboo/PBS composites with CMC 12.5g and PVA 12.5g shows higher than flexural strength of without additive. (3) In case of used CMC additive, the maximum value of $35.1 \mathrm{HV}$ of CMC $12.5 \mathrm{~g}$. Vickers hardness of bamboo/PBS composites used PVA additive. It can be seen highest value at $30.96 \mathrm{HV}$ case of PVA12.5g.

\section{Acknowledgments}

This work was supported by Electro Ceramic Center at Dong-eui University as a RIC program (2011) of ITEP under Ministry of Knowledge Economy and Busan Metropolitan City.

\section{References}

1. J. Jiang and T. Fujii, Reinforced Plastics 45, 365 (1999).

2. H. Takagi and R. Takura, Journal of the Society of Materials Science, Japan 52, (2003).

3. A.K. Mohanty, M. Misra and G. Hinrichsen, Biofibers 276, (2000).

4. S.Y. Lee, S.H. Hong, S.H Lee and S.J. Park, Macromol Biosci 4(3), 157 (1988).

5. K.A. Berglund, American Chemical Society 223, 669 (2002).

6. T. Willke and K.D. Vorlop, Appl Microbiol Biotechnol 66(2), 131 (2004).

7. Y. Liu, E. Ranucci, M.S. Lindblad and A.C. Albertsson, J.B.Compat Polym 17(3), 209 (2002).

8. E. Ranucci, Y. Liu, M.S. Lindblad, A.C. Albertsson. Macr Rapid Commun 21(10), 680(2000).

9. N. Kumar, R.S. Langer, A.J. Domb, Adv Drug Delivery Rev 54(7), 889(2002). 